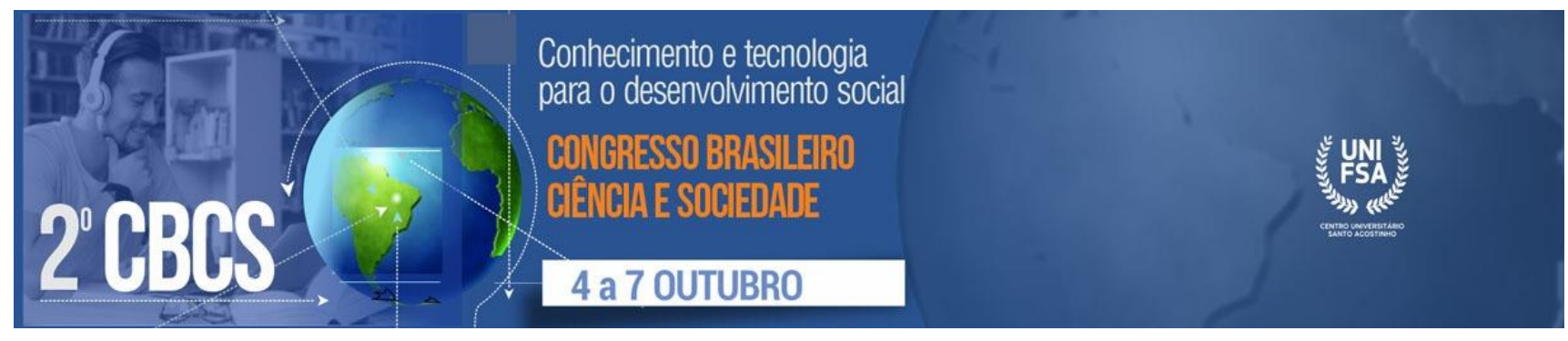

\title{
SAÚDE E APLICATIVOS MÓVEIS NO CONTEXTO DA COVID-19¹
}

\author{
Damião Pires de Sá/UESPI ${ }^{2}$ \\ Stálin Santos Damasceno/UESPI ${ }^{3}$ \\ Fabrício Pires de Moura do Amaral/UESPI ${ }^{4}$ \\ Dario Brito Calçada/UESPI ${ }^{5}$
}

\section{RESUMO}

Objetivo : Discutir artigos para obter uma ideia precisa e clara dos conhecimentos atuais sobre tema Dispositivos Móveis e Saúde no contexto da Covid-19. Métodos: Trata-se de revisão de artigos, pesquisados na base de dados Scopus com o descritor 'App and health and covid-19 and mobile devices', excluídos os anteriores ao mês de julho do ano de 2021, os repetidos, os relatos de casos, e os que não tratam diretamente do tema. Resultados: Dos 40 artigos encontrados na busca inicial, foram selecionados 5 artigos para leitura e análise minuciosa. Todos os artigos selecionados foram em língua inglesa, com publicações em países 5 diferentes: Luxemburgo, Brasil, Alemanha, Irã, e Estados Unidos. Considerações finais: Todos os profissionais precisam se atentar para as inovações que surgem e buscar conhecimentos para que possam produzir essas inovações ou no mínimo apreciá-las e utilizá-las da melhor forma possível para melhor atender a sociedade.

Palavras-Chave: Aplicativo, Saúde, Covid-19.

\section{INTRODUÇÃO}

Dentre os vários problemas enfrentados pela humanidade, a pandemia causada pelo vírus SARS-Cov-2, destaca-se como uma das mais preocupantes, no instante em que cria muitos desafios à promoção de saúde de forma local, e global. Esse trabalho busca discutir artigos para

\footnotetext{
${ }^{1}$ Trabalho apresentado no 20 Congresso Brasileiro Ciência e Sociedade (CBCS 2021), promovido pelo Centro Universitário Santo Agostinho, de 04 a 07 de outubro de 2021, em Teresina-PI.

${ }^{2}$ Damião Pires de Sá. Mestrando em Biotecnologia em Saúde Humana e Animal. Universidade Estadual do Piauí-UESPI, Brasil.

${ }^{3}$ Stálin Santos Damasceno. Especialista em Gestão em Saúde. Universidade Estadual do Piauí-UESPI, Brasil.

${ }^{4}$ Fabrício Pires de Moura do Amaral. Doutor em Farmacologia. Universidade Estadual do Piauí-UESPI, Brasil.

${ }^{5}$ Dario Brito Calçada. Doutor em Ciências da Computação e Matemática Computacional. Universidade Estadual do Piauí.
} 
obter uma ideia precisa e clara dos conhecimentos atuais sobre o tema Aplicativo e Saúde no contexto da Covid-19.

Quando se observa, por exemplo, que ao se estabelecer a avaliação de tecnologias na saúde, apesar de haver algumas experiências bem-sucedidas, elas de fato não fazem parte da gestão hospitalar da maioria das instituições de saúde do país, ou seja, as tecnologias ainda não são uma realidade de âmbito nacional nos estabelecimentos de saúde, o que demonstra o grande desafio que se tem pela frente quando se trata do uso de ferramentas para favorecimento da saúde no país (Francisco FR, et al 2019).

Nesse sentido, a conjuntura com desfecho de crise provocada pela pandemia necessita ser analisada e o aspectos holísticos da análise de desenvolvimento de aplicativos e da inovação na prática clínica e no contexto de uma crise de saúde precisam ser considerados. Para isso se procurou nos artigos aspectos relevantes relacionados a possível redução das fronteiras entre os sistemas de comunicação na saúde por meio do uso de dispositivos móveis, a inovação na comunicação da Covid-19, à gestão de equipamentos durante a pandemia de Covid-19 por meio desses instrumentos, e como aplicativos podem melhorar a qualidade de vida dos usuários de saúde.

\section{METODOLOGIA}

Trata-se de revisão de literatura, pesquisados na base de dados Scopus com o descritor 'App and health and covid-19 and mobile devices', excluídos os anteriores ao mês de julho do ano de 2021 , os relatos de casos, e os que não tratam diretamente do tema. Ao finalizar as pesquisas na base de dados, as referências repetidas foram excluídas. Foram selecionados apenas 5 dos 40 artigos encontrados inicialmente por meio dos descritores, escritos no idioma ingês. A coleta de dados foi realizada dos dias 24 de abril a 04 de maio de 2021.

Dos 40 artigos encontrados na busca inicial, foram selecionados 5 artigos para leitura e análise minuciosa. Todos os artigos selecionados foram em língua inglesa. As publicações foram escritas em 5 países diferentes: Luxemburgo, Brasil, Alemanha, Irã, e Estados Unidos. Após a leitura dos artigos selecionados, se observou a necessidade de se discutir cada vez mais a temática da Inovação e do uso de tecnologias como o Aplicativo na Saúde, principalmente, no contexto da Covid-19, no instante em que se faz doravante imprescindível incorporar cada vez mais na cultura organizacional dos profissionais de saúde a inovação, seja com o uso de 
tecnologias e dispositivos novos que tragam mais conveniência para os clientes e usuários, seja na forma dos profissionais atender as pessoas.

A pandemia da Covid-19 mostrou várias exigências de aspectos de inovação que não poderiam ser observáveis sem um acontecimento de caráter global, além de reforçar que o uso de aplicativos pode se tornar cada vez mais necessário no labor de todos os profissionais de saúde, da medicina, principalmente, no contexto de uma pandemia que exige distanciamento social, mas também uma redução das fronteiras entre os sistemas financeiros na saúde, conquistada por meio da inovação nos equipamentos, aplicativos e formas de gerir os recursos.

Os artigos demonstram ainda que os aplicativos aliados à inovação, podem melhorar o atendimento na saúde, no instante em que trazem mais conveniência e conforto para os usuários de saúde e permite que os profissionais possam avaliar melhor os riscos do paciente de forma a evitar riscos causados por equipamentos, erros de anotação, distribuição de recursos ou outros riscos externos.

Por outro lado, também se pode observar na leitura dos artigos que os investimentos de longo prazo que trazem inovações para a saúde e, consequentemente, todos esses benefícios no momento de uma pandemia como a Covid-19, muitas vezes, são substituídos por investimentos de curto prazo por que não deixam o capital parado, por exigência do mercado financeiro, e assim, impõe fragilidades ao desenvolvimento de aplicativos e da inovação em saúde, permitindo desinformações e até o uso incorreto de dispositivos tecnológicos.

Também se pode observar, que o uso de dispositivos móveis permite que os profissionais da saúde possam avaliar melhor os riscos do cliente, subsidiar decisões rápidas quando necessárias, comunicar com colegas de trabalho, clientes e usuários do sistema de saúde de forma rápida e mais confortável, de forma a evitar riscos causados por equipamentos, erros de anotação, distribuição de recursos, desinformações ou outros riscos ext ernos.

\section{DISCUSSÃO}

O fato de facilitar o acesso às informações, com conforto e praticidade nas soluções de mercados, inclusive relacionados ao trabalho, os dispositivos móveis se mostram convenientes no acesso sob demanda de informações e soluções de negócios de saúde, assim, os aplicativos móveis se tornaram um ativo importante no mundo digital (SAMHI, et al, 2021). 
No contexto da pandemia de COVID-19, desenvolvedores de aplicativos uniram esforços em busca de respostas para as mais diversas necessidades de serviços, e para os mais diversos usuários, e assim, passaram a oferecer muitos serviços, e informações, desde as mais genéricas até as mais especializadas, como as ajudas em diagnósticos. Nessa conjuntura, os aplicativos trouxeram algumas preocupações ao espalhar informações incorretas ou mesmo códigos e programas maliciosos (SAMHI, et al, 2021).

Assim, o estudo de Samhi e colaboradores, da Universidade de Luxemburgo, produziu uma série de percepções empíricas que contribuem para ampliar o conhecimento sobre os aplicativos relacionados à Covid, e demonstrou que comunidades de desenvolvedores contribuíram rapidamente para o combate a COVID-19, com aplicativos dedicados lançados no início de janeiro de 2020, que os aplicativos relacionados à Covid-19 fornecem ferramentas digitais aos usuários, como os usuários de saúde, que servem para transmitir informações aos usuários, inclusive informações estatísticas, para coletar dados dos usuários como, por exemplo, para rastreamento, que os aplicativos relacionados à Covid são menos complexos do que os aplicativos padrões, que eles geralmente não parecem vazar dados confidenciais, e que na maioria dos casos, os aplicativos relacionados à Covid são lançados por entidades com experiência anterior no mercado, principalmente entidades governamentais oficiais ou organizações públicas de saúde (SAMHI, et al, 2021).

A inovação e o uso de aplicativos também auxiliam na gestão de saúde, no instante em que, tanto podem melhorar a comunicação, o gerenciamento de leitos e outros equipamentos, podem reduzir custos como os de deslocamentos desnecessários, bem como dar uma visão mais precisa e holística em um menor espaço de tempo das unidades de saúde de uma determinada região, no instante em que podem servir como instrumento de medição de dados e como ferramenta de auxílio educacional ou de planejamento de estratégias de gestão em saúde (SALLES NETO, et al, 2020).

Ainda nesse contexto da pandemia de COVID-19, pesquisadores na Alemanha relataram que uma forma de reduzir futuras transmissões de SARS-CoV-2 é o uso generalizado de aplicativos de rastreamento de contatos, pois, esses aplicativos rastreiam os contatos próximos e avisam os contatos de pessoas com teste positivo para infecção. Assim, Blom e colaboradores, da Universidade de Mannheim, fizeram um estudo, em que analisaram as barreiras potenciais para a adoção em grande escala do aplicativo de rastreamento de contato oficial que foi introduzido na Alemanha em 16 de junho de 2020, assim, estimaram que 81\% da população de 
18 a 77 anos possui os aparelhos e a capacidade de instalar o aplicativo oficial e que $35 \%$ também estão dispostos a instalar e usar. Os estudiosos observaram ainda que os propagadores potenciais mostram alto acesso aos dispositivos necessários para instalar o aplicativo (92\%) e alta capacidade de instalar o aplicativo (91\%), mas baixa disposição (31\%) para adotar corretamente o aplicativo, enquanto para grupos vulneráveis, a principal barreira é o acesso (62\%) (Blom, et al, 2021).

Portanto, há uma visão pessimista sobre a eficácia do rastreamento de contato baseado em aplicativo para conter a pandemia de COVID-19, e se recomenda direcionar as campanhas de informação a grupos com alto potencial de propagação do vírus, mas que não desejam instalar e usar corretamente o aplicativo, em particular homens e pessoas com idade entre 30 e 59 anos, e grupos vulneráveis, em particular indivíduos mais velhos e aqueles em famílias de baixa renda, podem receber equipamentos e suporte para superar suas barreiras à adoção de aplicativos (Blom, et al, 2021).

A expansão dos aplicativos móveis de saúde para a gestão do COVID-19 cresceu exponencialmente nos últimos meses, entretanto, ainda são poucos os estudos que avaliaram esses dispositivos, assim, Salehinejad e colaboradores, da Universidade de Ciências Médicas de Kerman, no Irã, buscaram desenvolver uma medida confiável e classificar a qualidade dos aplicativos de saúde móveis COVID-19, para, eventualmente, fornecer um roteiro para o desenvolvimento futuro de aplicativos de Saúde. Este estudo sugeriu que a maioria dos aplicativos relacionados ao COVID atendem aos critérios aceitáveis de qualidade, conteúdo ou funcionalidade, e devem destacar recursos estéticos e interessantes para que a melhoria geral da qualidade seja bem-vinda pelos usuários (Salehinejad et al, 2021).

Steve Moeini e colaboradores, apresentaram um estudo de usabilidade de um aplicativo em duas fases: a fase inicial consistiu no design do aplicativo bruto desenvolvido para teste, e apresentou alguns problemas de usabilidade, já a segunda fase representou um refinamento sobre a fase inicial com algumas pequenas modificações no design do aplicativo. Assim, com base na resposta do usuário, a segunda fase foi bem-sucedida. As mudanças no aplicativo para os testes foram vitais para os usuários e receberam boas avaliações. Atividades podem ser aplicadas a pesquisas futuras com uma estratificação de usuários, como por faixa etária, para ver se há diferenças de tempo nos cenários de tarefas entre os grupos de idade (STEVE MOEINI, et al, 2021). 


\section{CONSIDERAÇÕES FINAIS/CONCLUSÕES}

Todos os profissionais precisam observar o mundo que os rodeia, se atentar para as inovações que surgem e buscar conhecimentos para que possam produzir essas inovações ou no mínimo apreciá-las e utilizá-las da melhor forma possível para melhor atender a sociedade que muitas vezes investiu bastante na formação desses profissionais. $\mathrm{O}$ uso da inovação aliada a aplicativos móveis permite que os profissionais da saúde possam avaliar melhor os riscos do cliente, subsidiar decisões rápidas quando necessárias, comunicar com colegas de trabalho, clientes e usuários do sistema de saúde de forma rápida e mais confortável, de forma a evitar riscos causados por equipamentos, erros de anotação, distribuiç̧ão de recursos, desinformações ou outros riscos externos.

É importante se lembrar que as inovações e desenvolvimento de aplicativos tem um custo, muitas vezes, alto. Mas, o conhecimento das novas tecnologias tem se tornado bastante acessível nos últimos anos. Não observar os problemas que a cercam é deixar de exercer a função de cidadania. Se se quer uma Sociedade mais justa e saudável, é imprescindível que cada profissional tenha bom conhecimento das inovações e dispositivos móveis que possam melhorar a vida das pessoas no que diz respeito a gestão, atuação e promoção da saúde.

\section{REFERÊNCIAS}

BLOM, A. G., et al. Barriers to the large-scale adoption of a covid-19 contact tracking application in Germany: research study. Journal of Medical Internet Research, 202123 (3) e23362.

Francisco FR, Malik AM. Application of health technology assessment (HTA) in the decisionmaking in hospitals. J Bras Econ Saúde 2019; 11:10-7.

SALEHINEJAD, S, et al. A review and content analysis of national applications for managing COVID-19 using the Mobile Application Rating Scale (MARS). Information Technology for Health and Social Assistance. Volume 46, Edição 1, 2021, Páginas 42-55.

SALLES NETO L. L., de, et al. Forecast UTI: application for forecasting beds in intensive care units in the context of the covid-19 pandemic. Epidemiol Serv Saúde [preprint]. 2020 [citado 2020 jul 28]:[13 p.]

SAMHI, J., et al. A first look at the Android apps on Google Play related to COVID-19. Empirical Software Engineering. Springer Nature, 2021. 26 (4) , 57.

STEVE MOEINI, et al. Development of a weighted well-being assessment mobile application for trauma-affected communities: a usability study. Perspect Health Inf Manag. 2021 Winter; 18 (inverno): 10. 